Journal of Accident and Emergency Medicine 1995 12, 23-27
Correspondence: D.I. Flitcroft, Opthalmology Senior House Officer. Western Eye Hospital, Marylebone Road, London NW1 5YE, UK

\title{
Who should see eye casualties?: a comparison of eye care in an accident and emergency department with a dedicated eye casualty
}

\author{
D.I. FLITCROFT ${ }^{1}$, M.WESTCOTT ${ }^{1}$, R.WORMALD ${ }^{1} \&$ R. TOUQUET ${ }^{2}$ \\ ${ }^{1}$ Western Eye Hospital, Marylebone Road, London and ${ }^{2}$ Department of Accident and Emergency Medicine, \\ St Mary's Hospital, London
}

\section{SUMMARY}

Emergency care for eye complaints is provided both by accident and emergency (A\&E) departments as well as by dedicated eye casualty departments. This study examines the role of each type of department and the quality of eye care provided. Significant differences were found between the accident and emergency department and the eye casualty department in the history, examination and management of eye patients. Most notably, there were significant differences in the quality of the assessment in the two institutions. Overall 19\% (19/100) of A\&E records had an inadequate history, compared with $2 \%(1 / 50)$ for eye casualty records. Fifty-nine per cent (59/100) of A\&E records contained a significant examination omission, compared with only $8 \%$ $(4 / 50)$ of eye casualty records. Most of the omissions related to a failure to perform an adequate, yet simple, ocular examination including failure to record visual acuity. In $44 \%(44-100)$ of A\&E cases visual acuity was not recorded or recorded incorrectly. In comparison acuity omissions in eye casualty were present in only $4 \%(2 / 50)$ of cases.

Key words: accident and emergency, casualty, opthalmology, primary care

\section{INTRODUCTION}

Ophthalmology is unusual amongst hospital-based specialties, in that many departments offer an open access service for patients with acute eye problems. Such eye casualty departments provide a primary care service in parallel to that offered by general practitioners (GPS) and A\&E departments. While the caseload and activity of dedicated eye casualty departments have been the subject of some scrutiny, ${ }^{1-3}$ only recently has attention been given to the ophthalmological role of A\&E departments. ${ }^{4,5}$ Direct comparison between such studies is made difficult by the varying locations of the study centres with the associated variations in population. A recent study ${ }^{6}$ has examined eye cases presenting to either GPs or to the local eye casualty department within a defined urban area. However, this study made no reference to attendances at A\&E departments.

The published studies have largely emphasized the demographic and diagnostic features of patients presenting to each institution. For A\&E departments, between 2.7 and $6.1 \%$ of new patients were found to have an eye-related problem and of these between 65 and $71 \%$ of cases were managed without referral to an ophthalmologist. ${ }^{4,5}$ Minor trauma was the most frequent cause for presentation. The most frequent attenders were males of working age. Only a single study $^{5}$ has directly addressed the question of the quality of eye care received by patients attending an A\&E department. While only $1 \%$ of cases were deemed to have been managed unsatisfactorily, significant omissions were revealed in the quality of the assessment with approximately one-third of patients having no documentation of visual acuity. Other aspects of the qual:ty of assessment of ocular conditions were not considered and no direct comparisons were made between standards in A\&E departments and in dedicated eye units.

This present study represents a collaboration between an A\&E department and an eye casualty department, which both offer a 24-h primary care service for eye complaints. The two institutions are situated less than a mile apart and therefore serve the same inner-city population. This provides an opportunity for direct comparison of the demographics and diagnostic categories of ocular accidents and emergencies in the two types of institution. Furthermore, it allows for direct comparison of the quality of the assessment and management of eye cases in the two types of facility.

\section{METHODS}

A review of case notes was performed for patients

(C) 1995 Blackwell Science Ltd 
presenting for the first time to either St Mary's A\&E Department or to the Eye Casualty Department at the Western Eye Hospital, both situated in west London and separated by 0.6 of a mile. The A\&E department was well equipped for ocular examination with Snellen charts, direct ophthalmoscopes, a slit lamp and routine topical ophthalmological preparations.

The study group comprised 50 patients who were initially assessed by SHOs in St Mary's A\&E department and referred to the on-call SHO ophthalmologist at the Western Eye Hospital during the study period, 50 randomly selected patients who presented to the A\&E department and were treated and discharged with no arrangement for ophthalmological review, and 50 randomly selected patients who attended the Western Eye Hospital as new patients. Those patients referred for ophthalmological review had separate eye casualty notes and, as a result, the total sample consisted of 200 case notes for 150 patients.

Each set of notes was marked by an experienced ophthalmologist according to a pre-defined score sheet that was compiled, after discussion with A\&E staff, taking into account the level of equipment available in the A\&E department. The score sheet recorded basic demographic data and adequacy of history noting specific omissions. Examination features were recorded in relation to the following pre-defined categories: acuity, lids, orbit, cornea, conjunctiva, anterior chamber, pupil (including relative afferent pupillary defect), fluorescein, fundoscopy, eye movements, fields and radiographs. Acuities in both eyes were recorded precisely as noted on the case notes (including any errors of notation). Diagnosis was recorded as stated in the notes and the case allocated to a general diagnostic category.

Any omissions were classified as being either significant or insignificant by the reviewing ophthalmologist. Omission of visual acuity was deemed to be significant in all cases, except where the eye had been enucleated (one case), whereas, for other features of the examination, the significance was determined in light of the nature of the presenting complaint.

\section{RESULTS}

\section{Nature of sample}

In the 6 months of the study 23130 patients registered at St Mary's A\&E Department of which 337 period, 50 ophthalmological referrals were made, which represents $15 \%$ of all eye cases. In comparison, the eye casualty department at the Western Eye Hospital had approximately 1800 patients attending for the first time over the 6-month period.

There was no statistically significant difference in the ages of the patients in the two institutions. The mean age of A\&E patients was 34.8 years, compared with 33.9 years for eye casualty patients. There was, however, a significant difference in the male:female ratio. In the A\&E department there was a predominance of males with a male:female ratio of 2.85:1 whereas at the eye casualty the male:female ratio was 1.08:1 $\left(\chi^{2}=7.26 ; \mathrm{df}=1 ; P<\right.$ 0.01).

\section{Documented history}

Overall 19\% (19/100) of the A\&E cards were deemed to have inadequate recorded histories. Most omissions related to the lack of recording of any visual symptoms. In cases of trauma, the mechanism of injury was generally adequately defined as was the time course of current symptoms. In contrast, the recorded history was deemed inadequate in only $2 \%(1 / 50)$ of the eye casualty cases $\left(\chi^{2}=8.34 ; d f=\right.$ $1 ; P<0.005)$.

\section{Ocular examination}

There were marked differences in the number of significant examination omissions between the two institutions. Overall $59 \%$ of A\&E notes contained one or more significant examination omissions (25/ 50 of non-referred cases and 34/50 of referred cases), compared with $8 \%(4 / 50)$ for eye casualty records $\left(\chi^{2}=35.6\right.$; $\left.\mathrm{df}=1 ; P<10^{-7}\right)$.

The nature of these omissions is shown in Fig. 1. This figure demonstrates firstly the predominance of omissions in recording visual acuity and also that other omissions are of a simple nature including the use of fluorescein and assessment of the cornea, eye movements and pupils. Within the A\&E department $25 \%$ of cases $(25 / 100)$ contained an error or an omission in visual acuity in isolation, 15\% (15/ 100) contained other examination omissions but with acuity correctly recorded and $19 \%(19 / 100)$ contained deficits in acuity and at least one other examination feature. Overall there was no significant association between acuity omissions and other examination deficits.

By far the most notable difference was the deficiency in recording visual acuity found in the A\&E 
Comparison of eye care

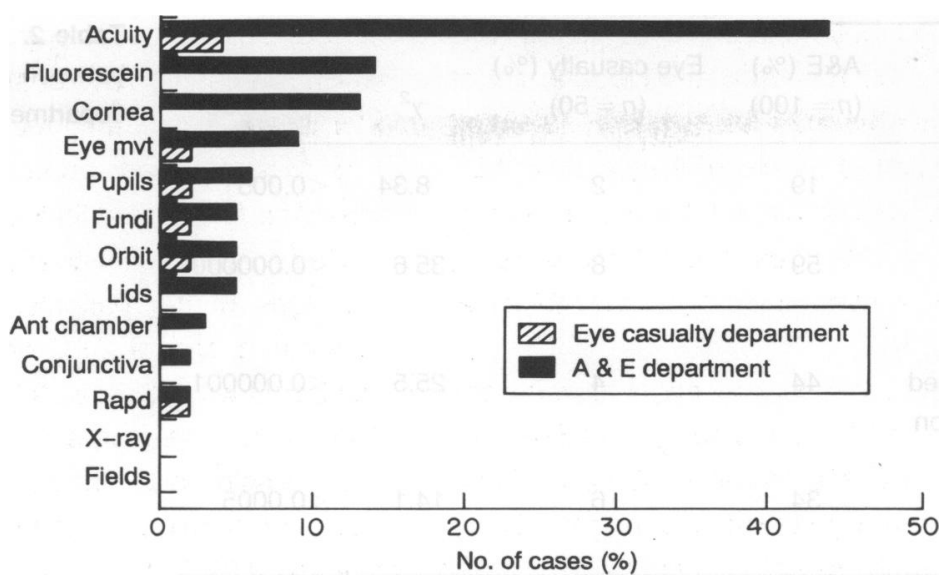

Fig. 1. Comparison of significant examination omissions in A\&E with significant examination omissions in the eye casualty department. department. Errors or omissions in recording visual acuity contributed to three quarters of the A\&E department cases with significant examination omissions. Overall, in the A\&E department, $44 \%$ (44/ 100 ) of cases either failed to record visual acuity or contained an error in recording acuity. In the eye casualty department the corresponding figure was only $4 \%(2 / 50)\left(\chi^{2}=25.5 ; \mathrm{df}=1 ; P<10^{-6}\right)$.

In $30 \%$ of the total sample from A\&E (19/50 non referred cases and 11/50 of referred A\&E cases), no form of acuity was noted at all. In a further sizeable proportion of accident and emergency cases the acuity was recorded incorrectly. This usually took the form of an inverted Snellen fraction, e.g. $18 / 6$. Such errors were present in $10 \%(5 / 50)$ of non referred $A \& E$ cases and $18 \%(9 / 50)$ of referred A\&E cases. There were no such errors in the eye casualty department.

To determine whether the visual acuity measurements recorded in the A\&E department corresponded to those measured at the eye casualty department, the acuity of the unaffected eye (in unilateral conditions) was examined in those patients that were referred to the eye casualty. As a result of to the frequent failure to record acuity in A\&E, only 22 patients had visual acuity recorded for an unaffected eye in both departments. Within this sample, $85 \%$ of the acuities recorded at the two sites matched to within one line of a Snellen chart and $95 \%$ matched within 2 lines. This degree of concordance suggests that there were no striking errors in terms of how acuity was measured in the A\&E department.

A similar number of examination features were recorded at both A\&E and the eye casualty department though there was a wide range at both institutions. In the eye casualty department a mean of 4.56 (SD 1.63) examination features were recorded per patient with a median value of 5 (range of 2 to 9). In the A\&E department the corresponding value for eye cases seen and discharged was 3.64 (SD 1.69) with a median of 4 (range 1 to 8 ). For A\&E patients referred on for ophthalmological review a mean of 4.8 (SD 1.75) and a median of five examination features were recorded per case (range 1 to 10). Overall in A\&E there was a mean of 4.22 (SD 1.82) and a median of four examination features recorded per patient (range 1 to 10 ).

\section{Diagnosis}

A wide variety of diagnoses were represented in the sample group, and in order to provide a more coherent description of the case mix, each case was placed into a diagnostic category as outlined in Table 1. Overall trauma constituted $51 \%(51 /$ $100)$ of caseload in A\&E with infection the second

\begin{tabular}{lccc}
\hline & $\begin{array}{c}\text { A\&E } \\
\text { not referred (\%) } \\
(n=50)\end{array}$ & $\begin{array}{c}\text { A\&E } \\
\text { referred (\%) } \\
(n=50)\end{array}$ & $\begin{array}{c}\text { Eye } \\
\text { casualty (\%) } \\
(n=50)\end{array}$ \\
\hline Trauma & 50 & 52 & 24 \\
Infection & 30 & 24 & 26 \\
Inflammation & 4 & 12 & 14 \\
Other & 2 & 12 & 16 \\
Normal & 8 & 0 & 12 \\
No diagnosis & 6 & 0 & 8 \\
\hline
\end{tabular}

Table 1. Caseload in A\&E and eye casualty departments by diagnostic category 
D.I. Flitcroft et al.

\begin{tabular}{lcccc}
\hline & $\begin{array}{l}\text { A\&E (\%) } \\
(n=100)\end{array}$ & $\begin{array}{c}\text { Eye casualty (\%) } \\
(n=50)\end{array}$ & $\chi^{2}$ & $P$-value \\
\hline $\begin{array}{l}\text { Deficient history } \\
\text { Significant exam }\end{array}$ & 19 & 2 & 8.34 & $<0.005$ \\
$\begin{array}{l}\text { omission } \\
\begin{array}{l}\text { Visual acuity omitted } \\
\text { or incorrect notation }\end{array}\end{array}$ & 44 & 8 & 35.6 & $<0.0000001$ \\
$\begin{array}{l}\text { Other significant } \\
\text { Exam omissions }\end{array}$ & 34 & 4 & 25.5 & $<0.000001$ \\
\hline
\end{tabular}

Table 2. Summary of differences between A\&E and eye casualty departments commonest category at $27 \%(27 / 100)$. At the eye casualty department a far wider variety of conditions were diagnosed with only $24 \%(12 / 50)$ represented by trauma. Comparing trauma with all other diagnoses, there was a significant difference between the patients in the eye casualty department as compared with A\&E ( $\left.\chi^{2}=9.98 ; \mathrm{df}=1 ; P<0.005\right)$.

By reviewing cases seen in the A\&E department that were referred on for ophthalmological review it was possible to assess directly the diagnostic accuracy within A\&E. Overall A\&E diagnoses tended to be less specific and more descriptive than those made at the eye casualty department. For patients referred to eye casualty there were 18 cases $(36 \%)$ where the diagnosis was different in the two sites. Glaucoma was diagnosed erroneously twice in A\&E. The most serious difference in diagnosis was a patient given a diagnosis of corneal abrasion in the A\&E department who was diagnosed as having a corneal ulcer at the eye casualty.

In three patients who were seen and discharged from the A\&E department, a serious ocular abnormality could not have been excluded safely on the basis of the recorded examination. Two of these cases related to periocular trauma and the third represented an undiagnosed red eye. The consequences of such cases in terms of subsequent morbidity or hospital reattendance are not known.

\section{Variation during the course of the study}

This study included a 5-month period during which none of the A\&E senior house officers (SHOs) changed jobs. We therefore looked for any indication that the quality of assessment changed over this period with increasing experience within the A\&E department. No such trend was apparent from the case notes from A\&E. This suggests that increasing any improvement in the quality of the recorded assessment.

\section{DIsCussion}

This study reveals significant differences between the quality of the recorded assessment in A\&E as compared with the neighbouring eye casualty department. While it is not surprising in itself that ophthalmology SHOs appear to offer better eye care for patients than SHOs in A\&E departments, the magnitude of the differences is remarkable (summarized in Table 2).

The marked differences in the rate of significant omissions between A\&E cases and eye casualty cases did not result from more comprehensive notes in the latter, as indicated by the similar number of examination features recored in both departments. The similarity in the number of recorded examination features suggests a failure within A\&E to select appropriate examination features. The examination omissions were also of a simple nature. Technically challenging tasks, such as fundoscopy, were far less frequently the subject of significant omissions, than simple aspects of examination of the anterior segment of the eye. The most important feature of the ocular examination, namely visual acuity, was the feature most regularly omitted in A\&E. Visual acuity provides vital diagnostic information and a reduction in acuity is one of the best indicators of serious ocular conditions. Failure to record visual acuity can, therefore, have potentially serious medicolegal implications.

The training and staffing levels in the A\&E department under study did not appear deficient. All the SHOs working in the A\&E department under study had attended a course in A\&E medicine that included a lecture on ophthalmological problems. Furthermore, they were also lectured during their tenure in 
Comparison of eye care the post by an ophthalmologist, and given instruction on the use of a slit lamp. Staffing levels were at those recommended by the Royal College of Surgeons, namely one SHO per 5000 new patients with no locum staff. The SHOs were also encouraged to seek help from A\&E registrars and consultants who were available in the department for approximately $16 \mathrm{~h}$ per day.

The training the SHOs had received was, on the basis of this study, either poorly retained or failed to emphasize the key features of ocular assessment. The variations in how information was recorded, the inclusion of irrelevant findings, and the omission of features of potential significance all point to a deficit in basic ophthalmological training. In light of the competing demands of other specialties it is suggested that ophthalmological training in A\&E should emphasize practical components of how to assess eye injuries on the basis of the range of conditions commonly seen in A\&E departments. A comprehensive diagnostic approach may need to be sacrificed to allow simpler messages concerning ocular assessment to be emphasized, not least the recording of visual acuity. It may have been that uncertainty between the medical and nursing staff as to who should record the acuity resulted in certain omissions. Having the nursing staff record the visual acuity when the patient arrives is a major advantage in that it allows consideration of visual acuity to influence triage priority. It is, however, ultimately the responsibility of the doctor to ensure that visual acuity has been recorded at some stage.

There are two contrasting interpretations of these findings. One interpretation is that, in view of differences in the quality of assessment of eye complaints in the two types of institution, all ocular accidents and emergencies should be seen by dedicated eye staff.
The alternative view is that since most of the omissions occurring in the A\&E department are of a simple nature, they should be remedied by improvements in training. The latter view has many advantages in that the necessary changes are cheaper. They also require no organizational change within the NHS. There would continue to be good accessibility to A\&E services for eye problems; a condition that could not be met by the smaller number of eye units. Furthermore, for junior doctors intending to become general practitioners, a post in A\&E will provide useful ophthalmological training and experience.

\section{REFERENCES}

1. Jones N.P., Hayward K.M., Khaw P.T., Clouaé C.M.P. \& Elkington A.R. (1986) Function of an ophthalmic accident and emergency department. Results of a six month survey. British Medical Journal 292, 188-190.

2. Chaipella A.P. \& Rosenthal A.R. (1985) One-year in an eye casualty clinic. British Journal of Ophthalmology 67, 865-870.

3. Vernon S.A. (1983) Analysis of all new cases seen in a busy regional centre ophthalmic casualty department during a 24 week period. Journal of the Royal Society of Medicine 76, 79-82.

4. Edwards R.S. (1987) Ophthalmic emergencies in a district general hospital casualty department. British Journal of Ophthalmology 71, 938-942.

5. Clancy M.J. \& Hulbert M. (1991) A Study of the eye care provided by an accident and emergency department. Archives of Emergency Medicine 8, 122-124.

6. Sheldrick J.H., Vernon S.A., Wilson A. \& Read S.J. (1992) Demand incidence and episode rate of ophthalmic disease in a defined urban population. (1992) British Medical Journal 305, 993-936. 